\title{
CORRESPONDENCE
}

\section{Asthma: surfactant eliminates the early allergen-induced response}

\section{To the Editor:}

In their recent article BABU et al. [1] have just published a remarkable finding in which they have clearly demonstrated how the inhalation of synthetic surfactant abolishes the early allergen-induced response in asthmatics. In interpreting these impressive results, however, they invoke theory based upon oedema at whose liquid-air interface surfactant could exert a "high surface pressure".

In pursuing this approach, the authors appear to have ignored a basic principle of surface physics. Forces derived from surface pressure/tension can only be translated into pressure differences for driving fluid $(\Delta P)$ if there is appreciable curvature to the interface as expressed by the Laplace equation, viz:

$$
\Delta P=\gamma\left\{\frac{1}{r_{1}}+\frac{1}{r_{2}}\right\}
$$

where $\gamma$ is the surface tension (reciprocating surface pressure) and $r_{1}$ and $r_{2}$ are radii of curvature in mutually perpendicular planes. Thus, respirologists focus much attention upon surface tension at the alveolar level where curvature is high $\left(r_{1}=r_{2} \cong 175 \mu \mathrm{m}\right), \Delta P$ amounting to as much as $8.0 \mathrm{~cm}$ water gauge (cm.w.g) if surfactant were not reducing the surface tension of water $\left(\gamma=69.9 \mathrm{mN} \cdot \mathrm{m}^{-1}\right)$. However, at the bronchial level $\left(r_{1}=1 \mathrm{~cm} ; r_{2}=\infty\right)$, the fluid pressure $(\Delta P)$ generated by pure water would be less than $0.07 \mathrm{~cm} . \mathrm{w} . \mathrm{g}$. Hence, even at its highest surface pressure $(\gamma \cong 0)$, surfactant could have no physiological effect whatsoever. Surfactant would simply cause the surface contribution to oedema formation $(\Delta P)$ to fall from one insignificant level to another.

It would therefore seem more likely that surfactant is acting upon the asthmatic lung by the alternative "barrier" mechanism, referenced by the authors [2], whereby surface-active phospholipid (SAPL) binding by adsorption to bronchial epithelium is "masking" irritant receptors which elicit the bronchoconstrictor reflex. However, this alternative approach now invokes the mechanism of adsorption of SAPL to solid surfaces, which conflicts with a belief, culturally embedded in respirology, that surfactant acts only at liquid-air interfaces. It is interesting that this "belief" also conflicts with the roles of SAPL in nonpulmonary sites in vivo and with vast experience in the physical sciences of surfactants studied at solid surfaces where they often form barriers [3].

Any role of surface tension/pressure by surfactant acting at the liquid-air interface of oedema must surely be a red herring, but it should not detract from a most exciting clinical finding.

\section{B.A. Hills}

Golden Casket Paediatric Research Laboratory, Mater Medical Research Institute, Brisbane, Queensland, Australia.

\section{References}

1. Babu KS, Woodcock DA, Smith SE, Staniforth JA, Holgate ST, Conway JH. Inhaled synthetic surfactant abolishes the early allergen-induced response in asthma. Eur Respir J 2003; 21: 1046-1049.

2. Hills BA. Asthma: is there an airway receptor barrier? Thorax 1996; 51: 773-776.

3. Hills BA. An alternative view of the roles of surfactant and the alveolar model. J Appl Physiol 1999; 87: 1567-1583.

\section{Role of interleukin-10 in idiopathic pulmonary fibrosis}

\section{To the Editors:}

We have read with great interest the article of BERGERON et al. [1], concerning the cytokine profile in tissues of patients with idiopathic pulmonary fibrosis (IPF). We strongly agree that finding out the roles of cytokines in IPF might be the key in understanding the pathogenesis of the disease, as well as inventing new therapy strategies for this foetal and unresolved disorder. We think that outlining the cytokine profile in the tissues of those patients is very important, even though, as the authors also mention, the number of patients included in their study (five) was unfortunately small.

We conducted a study in the University Hospital in Thessaly (central Greece), involving 20 patients with IPF and 11 patients with pulmonary fibrosis of a known cause, as well as 40 healthy volunteers, in whom we measured the serum levels of several cytokines, such as interleukin (IL)-2, IL-4, IL-8, IL-10 and interferon gamma. Interestingly, we found several differences between the serum levels of the two different patient groups, as well as those in patients and healthy volunteers. One striking result was that IL-10 was detected in increased levels in sera of patients with IPF, in comparison to healthy volunteers $(\mathrm{p}<0.05)$, and was not detected at all in patients with pulmonary fibrosis of a secondary cause. We have suggested the use of IL-10 in the differential diagnosis of patients with IPF, while BERGERON et al. [1], suggest it might be a possible therapeutic target for IPF.

We agree with the observation of BERGERON et al. [1], and think that IL-10 might be an important cytokine in IPF. Although the above observations have been made in both studies in a "given moment", the elevation in the amounts of IL-10 in both the tissues and serum of patients with IP, is an observation, which, we think, should not be ignored. Nevertheless, we assume that those "given moments" happened to be the same in both studies, since the serum and tissue samples have been obviously collected during the onset of the disease, before any treatment was administered. However, this was not very clear in the paper of BERGERON et al. [1], and we would like to know if it really happened as we assume.

The existing literature of observations concerning the IL-10 profile in pulmonary fibrosis is scarce. Most papers suggest that IL-10 has an antifibrotic effect, and derives from the 
alveolar macrophages [2], as well as from the pulmonary fibroblasts [3]. Its action is basically opposed to that of TNF- $\alpha$, which is a rather inflammatory cytokine [2]. It is considered, as well as IL-4, a major IL in the T-helper cell type 2 immune response [4]. Interestingly, IL-10 levels have been found to be high in rat tissues with pulmonary fibrosis, while in rats with IL-10 deficiency, the inflammatory response has been found to be more intense than in the wild animals [5]. In in vitro studies, the addition of IL-10 in fibroblasts has lessened the expression of type 1 collagen, the production of which is stimulated by transforming growth factor- $\beta$ [6]. Those observations support the findings of BERGERON et al. [1], as well those from our own studies, that IL-10 might be an important target for future therapies, as well as a diagnostic tool.

We think that investigating the interleukin-10 pathways and possible therapeutic uses might be important in discovering more about idiopathic pulmonary fibrosis and providing patients with a more effective treatment. Moreover, serum and tissue cytokine profiles might be important in this direction.

\section{P.G. Tsoutsou*, K.I. Gourgoulianis ${ }^{\#}$}

*Nikis 33, Syntagma, Athens and ${ }^{\#}$ Medical school, University of Thessaly, Larissa, Greece.

\section{References}

1. Bergeron A, Soler P, Kambouchner $\mathrm{M}$, et al. Cytokine profiles in idiopathic pulmonary fibrosis suggest an important role for TGF- $\beta$ and IL-10. Eur Respir J 2003; 22: 69-76.

2. Martinez JA, King TE, Brown K, et al. Increased expression of the interleukin-10 gene by alveolar macrophages in interstitial lung disease. Am J Physiol 1997; 273: 676-683.

3. Vancheri C, Mastruzzo C, Tomaselli V, et al. Normal human lung fibroblasts differently modulate interleukin-10 and interleukin-12 production by monocytes: implications for an altered immune response in pulmonary chronic inflammation. Am J Respir Cell Mol Biol 2001; 25: 592-599.

4. Zhou Y, Giscombe R, Huang D, Levfert AK. Novel genetic association of Wegener's granulomatosis with the interleukin 10 gene. J Rheumatol 2002; 29: 317-320.

5. Huaux F, Louahed J, Hudspith B, et al. Role of interleukin10 in the lung response to silica in mice. Am J Resp Cell Mol Biol 1998; 18: 51-59.

6. Arai $\mathrm{T}$, Abe $\mathrm{K}$, Matsuoka $\mathrm{H}$, et al. Introduction of the interleukin- gene into mice inhibited bleomycin- lung injury in vivo. Am J Physiol Lung Cell Mol Physiol 2000; 278: 914-922.

\section{From the authors.}

We thank P.G. Tsoutsou and K.I. Gourgoulianis for their interest in our manuscript in which we suggested a potential important role for transforming growth factor- $\beta$ and interleukin (IL)-10 in the pathogenesis of idiopathic pulmonary fibrosis (IPF) [1]. We also read with great interest the results they report concerning increased levels of IL-10 in the sera of their patients with IPF.
As they emphasised in their comments, the production of cytokines varies during the course of an inflammatory pulmonary process and it is very important to be precise about the moment of the study, which clearly corresponded in our work to "a given moment" of the development of IPF. All the tissue specimens studied were open lung biopsies obtained from patients with IPF at the time of histological confirmation of the diagnosis. It should be emphasised that no patient had a history or symptoms suggesting pulmonary fibrosis of a known cause and none had received any treatment for pulmonary fibrosis prior to lung biopsy.

In our study, we found that hyperplastic type II pneumocytes were the main source of IL-10 in the lung tissue specimens from patients with IPF [1]. One can speculate that at least a part of IL-10 produced in the lung in the course of IPF could circulate in the bloodstream, and thus could participate in the elevated levels of this cytokine in the sera of patients, as reported by P.G. Tsoutsou and K.I. Gourgoulianis. Additional work is needed, however, to determine the cellular sources of the increased circulating levels of IL-10 in IPF and to confirm that in the same patients, the serum and pulmonary levels of IL-10 are correlated.

The absence of increased levels of IL-10 in the blood of patients with known causes of pulmonary fibrosis raises concern about the histopathological pattern of the fibrotic process. Besides usual interstitial pneumonia, i.e. the histological counterpart of IPF, several other patterns have been identified in the spectrum of diffuse fibrotic interstitial pneumonias (such as desquamative interstitial pneumonia, nonspecific interstitial pneumonia, etc.) that can be associated with various aetiologies [2]. Cytokines involved in the pathogenesis of these patterns of pulmonary fibrosis might be different from cytokines present in IPF. Studies performed in situ on lung tissue samples from patients with secondary pulmonary fibrosis with precise histopathological pattern are needed to answer this question. In particular, the presence or absence and the extent of type II alveolar hyperplasia in these different interstitial pneumonias could also influence the amounts of IL-10 produced in the lung of these patients.

Finally, we agree with P.G. Tsoutsou and K.I. Gourgoulianis that studies evaluating the role of interleukin-10 in idiopathic pulmonary fibrosis may give further insights in the management of patients with this very disabling disorder.

\section{A. Tazi}

Service de pneumologie, Hôpital Avicenne, 125 rue de stalingrad, 93009 Bobigny, France.

\section{References}

1. Bergeron A, Soler $\mathrm{P}$, Kambouchner $\mathrm{M}$, et al. Cytokine profiles in idiopathic pulmonary fibrosis suggest an important role for TGF- $\beta$ and IL-10. Eur Respir J 2003; 22: 69-76.

2. ATS/ERS International multidisciplinary consensus classification of the idiopathic interstitial pneumonias. Am J Respir Crit Care Med 2002; 165: 277-304.

\section{Spirometry in young children}

\section{To the Editor:}

With interest we read the article by GRACCHI et al. [1]. In recent years we also evaluated the use of computer-animation programs for spirometry with children in our pulmonary function lab. However, we did not find a significant difference in maximal performance with or without the use of the "candles" and the "balloon" program. 